\title{
EDITORIAL
}

\section{Neonatal sepsis: need for consensus definition, collaboration and core outcomes}

\author{
Pediatric Research (2020) 88:2-4; https://doi.org/10.1038/s41390- \\ 020-0850-5
}

\section{OVERVIEW OF NEONATAL SEPSIS AND DEFINITIONS}

Sepsis represents a major contributor to global mortality and has been declared as a priority by the WHO. ${ }^{1}$ The highest sepsis incidence across all age groups is found in neonates affecting an estimated 3 million babies worldwide (22 per 1000 live births) with a mortality of $11-19 \%$ and unquantified long-term neurological defects. ${ }^{2,3}$

However, international data are difficult to standardise in the absence of unified criteria for neonatal sepsis. Recently, in adults, the Third International Consensus Definitions for Sepsis and Septic Shock (Sepsis-3) have defined sepsis as a life-threatening organ dysfunction caused by a dysregulated response to infection. ${ }^{4}$ The new consensus definition moved away from the concept of systemic inflammatory response syndrome, which formed part of the definition of sepsis in the past 20 years. Sepsis-3 criteria were developed and validated on large cohorts of electronic health record data-derived episodes from adults with sepsis. Despite the clear merits of the approach chosen by the Sepsis-3 taskforce, there are several pitfalls towards the translation of Sepsis-3 to neonates. The criteria to define infection and sepsis are essential in the neonatal population to limit overdiagnosis, but they are not part of the adult Sepsis-3 definitions. Sepsis-3 is based only on short-term outcomes but in neonates integration of predictors of long-term disability is critical. The criteria for organ dysfunction according to gestational and postnatal age need to be defined through systematic reviews and retrospective studies and validated in prospective studies.

The Sequential Organ Failure Assessment Score (SOFA) reflects changes in organ function altering from baseline. The pSOFA has been proposed and was found to be a reliable predictor of inhospital mortality in children. ${ }^{5}$ The recently described neonatal SOFA (nSOFA) predicted mortality on Very Low Birth Weight (VLBW) infants with late onset sepsis. ${ }^{6}$ In this issue of the journal an international group has provided an overview of the diverse definitions of neonatal sepsis with the aim of working towards international consensus.

\section{WHY NEONATES ARE DIFFERENT}

Neonates differ substantially to adults and older children due to altered immune function and potential intrauterine exposure to infection. ${ }^{7,8}$ The fetus is immune privileged in utero often resulting in endotoxin tolerance. This is altered by labour from a predominantly Th2 response to a more "adult" immune phenotype with an enhanced pro-inflammatory response. ${ }^{8,9}$ These differences are particularly prominent in preterm infants. However, neonatal immunology is not clearly delineated, and much has been extrapolated from research in umbilical cord blood that although easily accessible for study is more immunotolerant and does not entirely reflect postnatal immune responses. ${ }^{10}$ There are difficulties in determining the true risk of neonatal sepsis as the in utero environment cannot be easily assessed. For example, the duration of rupture of membranes and the presence of intrauterine infection are hard to diagnose with certainty. Placental pathology is likewise not entirely predictive and also not usually available at the time of sepsis evaluation. The lack of specificity of the majority of clinical signs and symptoms further complicate the identification of sepsis on the neonate.

Although Sepsis-3 concentrates on organ dysfunction in the diagnosis of sepsis, microbiological results are often still included in neonatal sepsis. Blood culture is only positive in approximately $0.5 \%{ }^{11,12}$ due to the small blood volume for blood cultures and antenatal maternal antibiotic use. There is no reliable single marker of sepsis and mortality and morbidity are high so empiric antibiotics are commenced in infants at risk. However, there is a need to balance the risk of morbidity and mortality from untreated infection versus the short- and long-term adverse effect of exposure to antibiotics. The risks of overuse of antibiotics are well-described in the era of antibiotic resistance and the negative effects of altering the microbiome include an increased rate of serious complications including mortality and necrotising enterocolitis association with antibiotic exposure.

In addition, neonatal sepsis is a heterogeneous condition, related to differences in gestational age, timing and source of infection. Coagulase-negative Staphylococci are often considered a contaminant or commensal in adults and older children but are associated with significant morbidity in preterm neonates, including adverse neurodevelopment. ${ }^{13}$ The difference in the NICU is that it may be more difficult to differentiate contamination from true infection and the long-term impact of these infections is greater on the developing brain. There are major differences between a baby arriving to the emergency room with a fever in the first month of life compared to a preterm infant born following severe chorioamnionitis and prolonged rupture of membranes. These varied populations of infants at risk of "neonatal sepsis" differ in many aspects of the disease, including clinical signs and symptoms, most likely pathogens and risk of mortality and longterm morbidity.

\section{CURRENT MARKERS OF NEONATAL SEPSIS}

Surrogate biomarkers of sepsis are commonly used due to the limitations of blood cultures alone to diagnose sepsis. Maternal infectious status is also important and placental pathology can provide a diagnosis of chorioamnionitis, although the relationship between histologic chorioamnionitis and neonatal sepsis is complex and ill-defined. Markers of systemic inflammation and immune responses include serial white cell counts and immatureto-mature granulocyte (IT) ratio. ${ }^{14-17}$ Serial full blood count values 
and IT ratios can predict the absence of early onset sepsis (EOS) with an AUC $\sim 0.8$ and negative predictive value for proven and suspected sepsis of $99 \%$ and $78 \%$, respectively. ${ }^{12}$ In addition, CRP and Procalcitonin demonstrate that biomarkers can be useful to shorten antibiotic treatment in patients who improve rapidly after treatment and have negative blood cultures. ${ }^{17-19}$ In addition, a recent meta-analysis and systematic review demonstrated that use of the neonatal EOS calculator is associated with a substantial reduction in the use of empirical antibiotics for suspected EOS. ${ }^{16}$

In view of the insensitivity of blood cultures alone to define neonatal infection, other techniques hold promise such as $16 \mathrm{~s}$ rRNA and PCR which detects $<3$ copies bacterium. Positives identified by PCR were higher than by blood culture (10 versus $5 \%)$ and when blood culture was used as control, the sensitivity and specificity of PCR was $100 \%$ and $97.85 \%$, respectively and the index of accurate diagnosis was $0.979 .{ }^{19}$ Multiplex PCR $(n=803$ infants and children) showed a positive test in $16 \%$ compared to $10 \%$ using blood culture. ${ }^{20}$ This is further improved in CSF samples increasing detection from 9 to $45 \%$. However, challenges still exist in the identification of clinically significant Gram-positive infections, understanding the significance of DNA of bacteria in the blood (DNAemia) and sustained inflammation on long-term outcomes.

\section{CONCLUSIONS}

The recent introduction of Sepsis-3 for adults has triggered plans to translate this to children and newborn infants and involvement in the Surviving Sepsis campaign (www.survivingsepsis.org). However, there are significant reasons that extrapolation is not appropriate in this setting. Sepsis is challenging for many reasons as it is not a single static disease but a dynamic continuum of inflammatory responses. This situation makes single biomarkers insufficient as different pathogens, immune status and duration of sepsis vary the systemic immune response.

Clinical trials have not routinely accounted for these variations and despite promise in defined subgroups have failed to prove benefit in larger populations. There are multiple definitions of neonatal sepsis used internationally that encompass clinical, microbiological and biochemical data as well as treatment initiation and duration. The difficulties in comparing early and late onset sepsis as well as differences between term and preterm infants make a single definition or management plan challenging. In EOS the presence of antenatal inflammation or chorioamnionitis may not be definitely recognised until the placental histology is completed and whether this information is included as a factor in early management is controversial. Histological confirmation of chorioamnionitis may or may not be helpful for the diagnosis of neonatal sepsis. ${ }^{21}$

More recently the neurodevelopmental sequelae of infection have been highlighted apart from the immediate morbidity and mortality. The inflammatory response following sepsis and necrotising enterocolitis are associated with adverse neurological outcomes. Even coagulase-negative Staphloccoccal infections that were previously considered as contaminants and harmless skin commensals are associated with abnormal neurodevelopmental outcome in preterm infants. ${ }^{22}$ Tertiary mechanisms of brain injury involve persistent dysregulated inflammation. Once inflammation is triggered, there can be a sustained response. ${ }^{23}$

In addition to the lack of an internationally accepted consensus definition of neonatal sepsis, there are no definitions associated with long-term outcomes. This lack hinders ongoing collaborative research and benchmarking. Core outcomes are required to standardise clinical trials of sepsis and allow comparison between trials. In addition, prioritising research goals with families is essential. ${ }^{24,25}$ Therefore a consensus definition is required that can be universally generalisable and validated in international datasets and correlated with neurodevelopmental outcomes.

\section{ACKNOWLEDGEMENTS}

This research was funded in part by the National Children's Research Centre, Dublin, Ireland. E.G. is supported by the Leenaards Foundation and E.J.M. by the Health Research Board of Ireland.

\section{AUTHOR CONTRIBUTIONS}

E.J.M.: Manuscript conception and design and wrote the manuscript. Literature review and manuscript revision. Revised and edited the manuscript before submission. M.M., E.G., H.K., M.A.T., A.v.d.H., J.B., J.M.K., F.M.K., J.M., M.D., S.H.P.S., W.P.deB., T.S., I.K.M.R., J.L.W., J.J., F.B.P., L.J.S.: Significant contributions to the intellectual content and literature review of the manuscript. Revised and edited manuscript before submission.

\section{ADDITIONAL INFORMATION}

Competing interests: The authors declare no competing interests.

Statement of original work: The authors confirm that this manuscript represents original work, has not been published previously and has not been submitted for publication elsewhere.

Publisher's note Springer Nature remains neutral with regard to jurisdictional claims in published maps and institutional affiliations.

Eleanor J. Molloy (iD ${ }^{1,2,3,4}$, James L. Wynn ${ }^{5}$, Joseph Bliss ${ }^{6}$, Joyce M. Koenig ${ }^{7}$, Fleur M. Keij ${ }^{8}$, Matt McGovern ${ }^{1,2^{\prime}}$ Helmut Kuester ${ }^{9}$, Mark A. Turner $^{10}$, Eric Giannoni ${ }^{11}$, Jan Mazela ${ }^{12}$ Marina Degtyareva ${ }^{13}$, Tobias Strunk ${ }^{14,15}$, Sinno H. P. Simons ${ }^{8}$, Jan Janota ${ }^{16}$, Franz B. Plotz ${ }^{17}$, Ages van den Hoogen ${ }^{18}$, Willem de Boode ${ }^{19}$, Luregn J. Schlapbach ${ }^{20,21}$ and

Irwin K. M. Reiss ${ }^{8}$ on behalf of the Infection, Inflammation, Immunology and Immunisation (14) section of the ESPR ${ }^{1}$ Paediatrics, Academic Centre, Children's Hospital Ireland (CHI) at Tallaght, Trinity College, the University of Dublin, Dublin, Ireland; ${ }^{2}$ Trinity Translational Medicine Institute, St James' Hospital, Dublin, Ireland; ${ }^{3}$ Neonatology, Coombe Women and Infants' University Hospital, Dublin, Ireland; ${ }^{4}$ Neonatology, CHI at Crumlin, Dublin, Ireland; ${ }^{5}$ Departments of Paediatrics \& Pathology, Immunology and Laboratory Medicine, University of Florida, Gainesville, FL, USA; ${ }^{6}$ Department of Pediatrics, Women \& Infants Hospital of Rhode Island, Alpert Medical School of Brown University, Providence, RI, USA; ${ }^{7}$ Division of Neonatology, Saint Louis University, Edward Doisy Research Center, St. Louis, MO, USA; ${ }^{8}$ Department of Pediatrics,

Division of Neonatology, Erasmus MC-Sophia Children's Hospital,

Rotterdam, The Netherlands; ${ }^{9}$ Neonatology, Clinic for Paediatric Cardiology and Intensive Care, University Medical Centre Göttingen, Göttingen, Germany; ${ }^{10}$ Institute of Translational Medicine, University of Liverpool, Centre for Women's Health Research, Liverpool Women's

Hospital, Liverpool, UK; ${ }^{11}$ Childrens Hospital Clinic of Neonatology, Department Mother-Woman-Child, Lausanne University Hospital and University of Lausanne, Lausanne, Switzerland; ${ }^{12}$ Department of Neonatology and Infectious Diseases, Poznan University of Medical Sciences, Poznan, Poland; ${ }^{13}$ Neonatology, Pirogov Russian National Research Medical University, Moscow, Russia; ${ }^{14}$ Neonatal Health and Development, Telethon Kids Institute, Perth, WA, Australia; ${ }^{15}$ Neonatal Directorate, King Edward Memorial Hospital for Women, Perth, WA, Australia; ${ }^{16}$ Department of Neonatology, Thomayerova nemocnice, Prague, Czechia; ${ }^{17}$ Department of Pediatrics, Tergooi Hospital, Blaricum, The Netherlands; ${ }^{18}$ Neonatology, Wilhelmina Childrens Hospital, University Medical Center, Utrecht, The Netherlands; ${ }^{19}$ Radboud Institute for Health Sciences, Department of Neonatology, Radboud University Medical Center, Amalia Children's Hospital, Nijmegen, The Netherlands; ${ }^{20}$ Paediatric Critical Care

Research Group, Child Health Research Centre, University of Queensland, Paediatric Intensive Care Unit, Queensland Children's Hospital, Brisbane, QLD, Australia and ${ }^{21}$ Department of Pediatrics,

Bern University Hospital, University of Bern, Bern, Switzerland Correspondence: Eleanor J. Molloy (eleanor.molloy@tcd.ie) 


\section{REFERENCES}

1. Reinhart, K. et al. Recognizing sepsis as a global health priority-a WHO resolution. N. Engl. J. Med. 377, 414-417 (2017).

2. Schlapbach, L. J. et al. Impact of sepsis on neurodevelopmental outcome in a Swiss National Cohort of extremely premature infants. Pediatrics 128, e348-e357 (2011).

3. Fleischmann-Struzek, C. et al. The global burden of paediatric and neonatal sepsis: a systematic review. Lancet Respir. Med. 6, 223-230 (2018).

4. Singer, M. et al. The third international consensus definitions for sepsis and septic shock (sepsis-3). JAMA 315, 801-810 (2016).

5. Matics, T. J. \& Sanchez-Pinto, L. N. Adaptation and validation of a pediatric sequential organ failure assessment score and evaluation of the sepsis-3 definitions in critically ill children. JAMA Pediatr. 171, e172352 (2017).

6. Wynn, J. L. \& Polin, R. A. A neonatal sequential organ failure assessment score predicts mortality to late-onset sepsis in preterm very low birth weight infants. Pediatr Res. https://doi.org/10.1038/s41390-019-0517-2 (2019).

7. Zonneveld, R. et al. A reevaluation of soluble adhesion molecules as markers for sepsis and the potential pathophysiological discrepancy in neonates, children and adults. Crit. Care 18, 204 (2014).

8. Zhang, X., Zhivaki, D. \& Lo-Man, R. Unique aspects of the perinatal immune system. Nat. Rev. Immunol. 17, 495-507 (2017).

9. Wolfs, T. G. et al. Inflammation-induced immune suppression of the fetus: a potential link between chorioamnionitis and postnatal early onset sepsis. J. Matern. Fetal Neonatal Med. 25, 8-11 (2012). Suppl 1.

10. Olin, A. et al. Stereotypic immune system development in newborn children. Cell 174, 1277-1292 (2018).

11. Escobar, G. J. et al. Stratification of risk of early-onset sepsis in newborns $\geq$ 34 weeks' gestation. Pediatrics 133, 30-36 (2014).

12. Mikhael, M., Brown, L. S. \& Rosenfeld, C. R. Serial neutrophil values facilitate predicting the absence of neonatal early-onset sepsis. J. Pediatr. 164, 522-528.e13 (2014).

13. Stoll, B. J. et al. Neurodevelopmental and growth impairment among extremely low-birth-weight infants with neonatal infection. JAMA 292, 2357-2365 (2004).
14. Chirico, G., Gasparoni, A., Ciardelli, L., Martinotti, L. \& Rondini, G. Leukocyte counts in relation to the method of delivery during the first five days of life. Biol. Neonate. 75, 294-299 (1999).

15. Wiland, E. L. et al. Adult and child automated immature granulocyte norms are inappropriate for evaluating early-onset sepsis in newborns. Acta Paediatr. 103, 494-497 (2014).

16. Achten, N. B. et al. Association of use of the neonatal early-onset sepsis calculator with reduction in antibiotic therapy and safety: a systematic review and metaanalysis. JAMA Pediatr. https://doi.org/10.1001/jamapediatrics.2019.2825 (2019).

17. Benitz, W. E. Adjunct laboratory tests in the diagnosis of early-onset neonatal sepsis. Clin. Perinatol. 37, 421-438 (2010).

18. Agyeman, P. K. A. et al. Epidemiology of blood culture-proven bacterial sepsis in children in Switzerland: a population-based cohort study. Lancet Child Adolesc. Health 1, 124-133 (2017).

19. Shang, S., Chen, G., Wu, Y., Du, L. \& Zhao, Z. Rapid diagnosis of bacterial sepsis with PCR amplification and microarray hybridization in $16 \mathrm{~S}$ rRNA gene. Pediatr. Res. 58, 143-148 (2005).

20. Lucignano, B. et al. Multiplex PCR allows rapid and accurate diagnosis of bloodstream infections in newborns and children with suspected sepsis. J. Clin. Microbiol. 49, 2252-2258 (2011).

21. Higgins, R. D. et al. Evaluation and management of women and newborns with a maternal diagnosis of chorioamnionitis: summary of a workshop. Obstet. Gynecol. 127, 426-436 (2016).

22. McGovern, M., Flynn, L., Coyne, S. \& Molloy, E. J. Does coagulase negative staphylococcal sepsis cause neurodevelopmental delay in preterm infants? Arch. Dis. Child. 104, 97-100 (2019).

23. Fleiss, B. \& Gressens, P. Tertiary mechanisms of brain damage: a new hope for treatment of cerebral palsy? Lancet Neurol. 11, 556-566 (2012).

24. Molloy, E. J. et al. Developing core outcome set for women's, newborn, and child health: the CROWN Initiative. Pediatr. Res. 84, 316-317 (2018).

25. Molloy, E. J., Mader, S., Modi, N. \& Gale, C. Parent, child and public involvement in child health research: core value not just an optional extra. Pediatr. Res. 85, 2-3 (2019). 